Verweildauer

\title{
Altenpfleger bleiben ihrem Beruf treu
}

— „Pflegeberufe nehmen einen Spitzenplatz bei der Verweildauer im Beruf ein! Wer ständig erzählt, Altenpflegerinnen und Altenpfleger würden nach fünf bis sieben Jahren aus ihrem erlernten Beruf aussteigen, kann entweder nicht rechnen oder legt

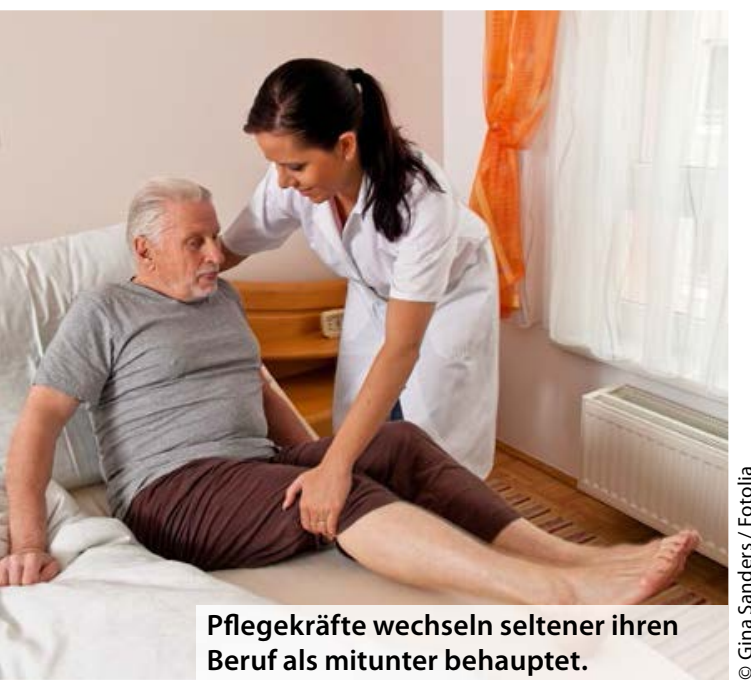

böswillig die Axt an den Ruf eines gesamten Berufsstandes", meldet sich Bernd Meurer, Präsident des Bundesverbandes privater Anbieter sozialer Dienste (bpa), zu Wort. „Denn das hieße, dass die Branche mit fast einer Million Beschäftigten alle fünf bis sieben Jahre komplett ihr Personal auswechseln würde. Mit Verlaub: Das ist Unsinn."

Die unabhängig erhobenen Zahlen des Bundesinstitutes für Berufsbildung (BiBB) seien völlig andere und müssten auch von Politikern zur Kenntnis genommen werden. Erwerbspersonen, die einen Pflege- oder Gesundheitsberuf erlernt haben, wechselten im Vergleich zu anderen Berufsfeldern deutlich seltener ihren Beruf. 2013 lag die sogenannte Stayer-Quote (Verbleib derjenigen, die im erlernten Beruf auch tatsächlich tätig sind) bei $74,4 \%$. Dies sei im Vergleich mit der durchschnittlichen Stayer-Quote von etwa $46,7 \%$ ein sehr hoher Wert.

www.bpa.de
Hausengel PflegeAllianz Besser kooperieren

— Immer mehr Pflegebedürftige in Deutschland werden im häuslichen Umfeld versorgt. Doch häufig mangelt es neben fehlenden häuslichen Strukturen an ausreichend auf- und miteinander abgestimmten Dienstleistern. Hier will sich die Hausengel PflegeAllianz künftig stark machen: Der gemeinnützige Verein hat sich auf die Fahne geschrieben, die regionale Pflege und Versorgung zu verbessern. Vorstandsvorsitzender Markus Oppel hat sich Fachleute an die Seite geholt: Pflegefachkräfte, pflegende Angehörige, private Dienstleister aus der ambulanten Versorgung wie auch Betreuungskräfte aus Osteuropa. Sie wollen erreichen, dass vor Ort noch verzahnter und effektiver zusammengearbeitet wird. Der Ursprung der Allianz liegt im „Bundesverband der Betreuungskräfte e.V. (BDBK)“. Als bislang einziger Verband deutschlandweit vertrat dieser die Interessen tausender, osteuropäischer Betreuungskräfte.

www.hausengel-pflegeallianz.de

\section{DPR: Generalistische Pflegeausbildung kommt zumindest scheibchenweise}

— Der Deutsche Pflegerat (DPR) bedauert das Scheitern der großen Reform der Pflegeausbildung. „Den zwischen den Koalitionsfraktionen jetzt gefundenen Kompromiss zum Pflegeberufereformgesetz sieht der DPR als ersten Schritt einer Reform an, auch wenn die drei Berufsabschlüsse erhalten bleiben", kommentiert DPR-Präsident Andreas Westerfellhaus. „Für die Krankenpflege ist es ein größerer, für die Alten- und Kinderkrankenpflege leider aber nur ein kleiner Schritt, um die Pflegeberufe zukunftssicherer zu machen und damit die Patientensicherheit zu gewährleisten." Die generalistische Pflegeausbildung komme nun zumindest scheibchenweise.

In der die Ausbildung zur Krankenpflege ablösenden generalistischen Pflegeausbildung werden laut DPR künftig die Stärken und Besonderheiten der drei bisherigen Pflegeberufe gebündelt. „Das ist der weiterhin richtige Ansatz und gewährleistet, dass unabhängig davon, an welchem Ort der Patient behandelt wird, das nötige pflegerische und medizinisch orientierte Wissen umfassend vorhanden ist", heißt es. Die hochschulische Ausbildung als zweiter Zugang zum Beruf und die vorbehaltenen Aufgaben seien wichtige Meilensteine. Westerfellhaus weiter: "Mit Blick auf die Alten- und Kinderkrankenpflege hätten wir uns dagegen wesentlich mutigere Schritte der Koalitionsfraktionen des Deutschen Bundestages gewünscht." Hier bleibe der Kompromiss weit hinter dem zurück, was Zielsetzung des Pflegeberufereformgesetzes ist. Es stehe zu befürchten, „dass es vor allem für die stationäre Altenpflege perspektivisch zu einem Absinken der Ausbildungszahlen kommen wird", so der DPRPräsident.

www.deutscher-pflegerat.de

\section{Alles richtig?}

Die korrekten Antworten der PflegeKollegs lauten:

Allergien + Unverträglichkeiten

$1 c, 2 c, 3 a, 4 b, 5 c, 6 a, 7 b, 8 c, 9 b, 10 b$

Aus Fehlern lernen

1a, 2c, 3b, 4c, 5a, 6b, 7c, 8a, 9c, 10a

Vorschau! Die PflegeKollegs im Monat Juni:

Leben mit HIV

Therapie - Pflege - Ernährung

Stark am Markt

Gut positionieren - Krisenfall managen - Außenwirkung verbessern 